\title{
A Comparitive Study of the Level of Psychological Distress of Female Cardiovascular and Cancer Patients
}

\author{
Dr. Roshan Anie Alex \\ Post Doctoral Fellow, Department Of Psychology, Kerala University, Kerala, India.
}

\begin{abstract}
The study was taken up to probe the level of psychological distress of female cardiovascular and cancer patients. The sample for the present study consisted of 225 women from Kerala, which consist of 75 cardiovascular ( $c v d$ ) patients, 75 cancer patients selected using purposive sampling technique from the various hospitals across Kerala and a comparative group of 75 normals selected from the general population. Cvd patients, cancer patients and normals showed significant differences in their level of psychological distress. The findings showed that Cvd patients possessed significantly higher level of depression, anxiety and psychological distress when compared to cancer patients and normals. Cancer patients possessed significantly higher level of stress than cvd patients. Normals had significantly higher level of stress than both the patient groups. It is hoped that the outcome of this study will lead to identify the level of psychological distress of female cvd and cancer patients.
\end{abstract}

Keywords: Psychological distress, cardiovascular disease, cancer.

\section{Introduction}

Lifestyle diseases are increasing in India, and are emerging as a serious cause of death. Recent studies report that cardiovascular diseases and cancer are increasing in women. Women who play multiple roles face a lot of psychological stress and strain and ultimately result in these disorders. The increasing emphasis on the interaction between psychological factors and development of disease is attracting considerable attention. The relationship between the personality of an individual, perception of stress and disease has long intrigued both the general public and researchers in the field of Psychology and Medicine.

It is evident that from the literature reviewed that psychological factors have a role in the development and prognosis of life diseases. Substantial psychological distress has frequently been observed in people with chronic Illness. Psychological distress in chronic illness has been shown to be independently associated with increased symptom report, disability and the utilization of health care resources (Lecrubier, 2001). Major depression in people who have suffered a myocardial infarction (MI) has been reported to be around 15 to $20 \%$, with an additional $27 \%$ reporting symptoms of minor depression. The prevalence of depression and anxiety is high in both chronic obstructive pulmonary disease (8-80\% depression; 6-74\% anxiety) and chronic heart failure (10-60\% depression; $11-45 \%$ anxiety). Co-morbid depression and anxiety are associated with increased mortality and healthcare utilisation and impact upon functional disability and quality of life (Yohannes et al., 2010). The greatest proportion of psychological distress (10\%) was estimated among participants with selfreported chronic heart failure (CHF). Perhaps most alarming is the very low number of patients who saw a mental health professional within the past year, which was only 35\%, even in those heart failure (HF) patients with psychological distress. Almost $40 \%$ of the participants reported depressive symptoms. Not more than $8 \%$ of these $388 \mathrm{HF}$ patients with depressive symptoms had anti-depressive medication described at discharge from the hospital (Rasul et al., 2005). Similarly, in people with cancer, depression as a comorbid syndrome has been estimated to affect 15 to $25 \%$ of patients although rates of over $40 \%$ have been reported for certain malignancies (Chochinov, 2005). The percentage of cancer patients with an anxiety disorder has been estimated to range from less than $1 \%$ to around $50 \%$ and similar figures have also been reported for psychological distress (Van'tSpijker et al., 1997). The prevalence rates for psychological distress among cancer patients have been in the range of 25 to $30 \%$ (Zabora et al., 2001).

1.2 Gender related differences in cardiovascular diseases and cancer.

The disease pattern, types of cardiac disorders and cancer and the physical, psychological, and environmental factors leading to these diseases of both men and women differs. The incidence of heart disease is more prevalent in men compared to women. However, it can be stated that heart disease is not just a man's disease. Heart attack, stroke and other cardiovascular diseases are devastating to women too. While most middle aged heart attack victims are men, heart attacks that occur in later life are the principle cause of death for both men and women. Heart disease is the leading cause of death in women after the age of 66, in men it is the leading killer beginning at the age of 39. Women compared to men, often have chest pains for a long time before a heart attack, in men, such pains more often mean a heart attack has already begun (The New Indian 
Express, 2009).Women experience a higher risk of cardiovascular disease after menopause. They typically gain weight during menopause and this weight gain may partly explain the enhanced risk, increases in blood pressure, cholesterol and triglycerides also risk factors for CHD (Wing, Matthews, Kuller, Meilahn and Plantinga, 1991), may also occur. We now know cardiac diseases are not just a man's problem. It is recognized that there are gender differences regarding the disease presentation, occurrence of more severe disease in women in certain age groups, differences regarding prevalence related to age, which will affect co-morbidity and there are also findings suggesting that the underlying mechanisms of disease might be gender-specific. Thus, gender-related differences in the lipid profile, hormonal status and influence of menopause, body composition, etc. might make the clinical presentation, the interpretation of the diagnostic findings and prognosis of similar clinical conditions to differ between sexes (Kesteloot et al., 2006).

Although some differences are hormonally related, there are probably many more differences between the sexes that are caused by behaviour rather than sex hormones. Although there are cases in which the incidence of cancer is higher in women, there are far more in which the men outstrip the women, largely because of different smoking and drinking behaviours, and in some cases, occupational exposures. Stomach cancer is related presumably to a combination of things that create reflux, like late night eating, drinking of alcohol and coffee, and smoking. For liver cancer, the incidence in men is estimated at about 10,000 versus 5,300 in women, again probably related to more alcohol consumption, which also accounts for the greater incidence of head and neck cancers in men. Meningioma, a type of brain cancer, makes up about $15 \%$ of brain tumors. It is much more common in women, probably because this particular tumor has estrogen and progesterone receptors. For that reason, tamoxifen is sometimes part of the treatment for this kind of brain tumor. It illustrates that even though some cancers are not thought to be related to the sex of the patient, the hormonal environment is indeed relevant. Malignant tumors of the thyroid are much more common in women than in men. Although the reason for this is not clearly spelled out, it is known that women have more autoimmune disease than men, and this could explain some of the difference. Women are also much more likely to get cancer of the gallbladder - about 4,000 new cases in women versus 2,900 in men. Women who are overweight are at higher risk, and historically women are more overweight than men. Differences in the metabolism of steroids and cholesterol in men and women is related in part to the estrogen and androgen difference. More men than women get squamous cell cancer of the lungs - the kind associated with smoking than do women. Some studies have shown gender differences at the cellular level, with relatively high aromatic hydrophobic DNA adducts in female as opposed to male lung cancer patients. Hormonal factors, such as late age at menopause and estrogen replacement therapy, may also play a role in greater female susceptibility to tobacco carcinogens (Michaela Kreuzer, 2002).

Women are not included as research subjects in studies of many major disease because cyclical variations in hormones are thought to obscure results or because pharmaceutical companies fear lawsuits if experimental drugs have adverse effects on women of childbearing age, harming foetuses or putting future children at risk (Taylor et al., 2000). Heart disease research has been based largely on men, and women are often ignored in studies of cancers, except for reproductive cancers. It is essential to include women in medical studies for many reasons (Mathews, Shumaker et al., 1997). First, women may have different risk factor for major diseases, or existing risk factors may be more or less virulent. Men and women differ in their biochemistry, and they differ in their physiological reactions to stress (Baum and Grunberg, 1991). Consequently, their symptoms may be different, their age of onset for the same diseases may differ and their reactions to treatment and needed dosage levels of medications may be different. For example, women's risk for coronary heart disease increases greatly following menopause. Until recently, however, research unearthing these important relationships is not even conducted. Without a systematic investigation of women's health and their particular risk factors, as well as changes in both over the life span, women will simply be treated more poorly than men for the same diseases.

Having noticed such differences between men and women regarding cardiovascular disorders and cancer, this study is taken up to probe the psychological distress of female cardiovascularand cancer patients. Though, this is of great relevance, the investigator was not able to trace studies done in the Kerala context. This prompted the investigator to take up a study of this nature, focusing on the women in Kerala state in India, with a view to find out the level of psychological distress in female cardiovascular and cancer patients.

\section{Methodology}

2.1. Sample: The sample for the present study consisted of 225 women from Kerala, which consist of 75 cardiovascular patients, 75 cancer patients, and 75 normals. The cvd patients and cancer patients were selected using purposive sampling technique from the various hospitals across Kerala. Comparable group of normals were selected from the general population. Cardiovascular disease types selected for the study were coronary artery disease, cardiomyopathy, aneurysm, myocardial infarction, ischemic heart disease. The cancer types were breast cancer, ovarian cancer, lung cancer, colorectal cancer, thyroid cancer and cervical cancer. 
2.2. Tools: The tools used for collecting the data were Personal data schedule and Depression Anxiety Stress scale (DASS 21). The Depression Anxiety Stress Scales 21 (DASS-21) developed by Lovibond, S.H and Lovibond, P.F is a set of three self-report scales designed to measure the negative emotional states of depression, anxiety and stress. Each of the three DASS 21 scales contains seven items, divided into subscales with similar content. The Depression scale assesses dysphoria, hopelessness, devaluation of life, self-deprecation, lack of interest / involvement, anhedonia, and inertia. The Anxiety scale assesses autonomic arousal, skeletal muscle effects, situational anxiety, and subjective experience of anxious affect. The Stress scale is sensitive to levels of chronic non-specific arousal. It assesses difficulty relaxing, nervous arousal, and being easily upset/agitated, irritable/over-reactive and impatient. The reliabilities of the DASS21 scales were .88 for Depression, .82 for Anxiety, .90 for Stress, and .93 for the Total scale. The validities of the DASS- 21 for depression, anxiety and stress subscales were 337, 328 and 347, respectively.

2.3. Data collection procedure: Data were collected individually after ascertaining the willingness and cooperation on the part of the respondents. Incomplete response sheets were not scored and used for analysis.

2.4. Statistical techniques: The statistical techniques used for analyzing the data were one-way ANOVA and Duncan test.

\section{Results And Discussion}

The scores obtained by the three groups under study (cvd patients, cancer patients and normals) on DASS were analyzed using one way ANOVA to find out whether there were any significant difference among the three groups on the level of depression, anxiety, stress and total psychological distress. The details are given in Table No.1.

Table No.1One way ANOVA of the scores obtained on depression, anxiety, stress and total psychological distress among the three groups- cardiovascular patients, cancer patients and normals

\begin{tabular}{|c|c|c|c|c|c|}
\hline Variable & Source & $\begin{array}{c}\text { Sum of } \\
\text { Squares }\end{array}$ & Df & Mean Square & F ratio \\
\hline \multirow{3}{*}{ Depression } & Between Groups & 3645.662 & 2 & 1822.831 & \multirow{3}{*}{$59.552 * *$} \\
\hline & Within Groups & 6795.227 & 222 & 30.609 & \\
\hline & Total & 10440.889 & 224 & & \\
\hline \multirow{3}{*}{ Anxiety } & Between Groups & 208.640 & 2 & 104.320 & \multirow{3}{*}{$4.916^{* *}$} \\
\hline & Within Groups & 4711.360 & 222 & 21.222 & \\
\hline & Total & 4920.000 & 224 & & \\
\hline \multirow{3}{*}{ Stress } & Between Groups & 530.169 & 2 & 265.084 & \multirow{3}{*}{$12.015^{* *}$} \\
\hline & Within Groups & 4898.027 & 222 & 22.063 & \\
\hline & Total & 5428.196 & 224 & & \\
\hline \multirow{3}{*}{$\begin{array}{c}\text { Total } \\
\text { Psychological } \\
\text { Distress }\end{array}$} & Between Groups & 2504.960 & 2 & 1252.480 & \multirow[b]{3}{*}{$16.861 * *$} \\
\hline & Within Groups & 16491.200 & 222 & 74.285 & \\
\hline & Total & 18996.160 & 224 & & \\
\hline
\end{tabular}

Note: ** the $\mathrm{F}$ ratio is statistically significant at 0.01 level

The F ratios indicated that there were significant differences in the level of depression, anxiety, stress and total psychological distress among cvd patients, cancer patients and normals. Duncan's Multiple Range test provides different critical difference values for particular comparisons of means depending on how adjacent the means are. Duncan test was done to find the significance of differences among the three groups on their level of depression, anxiety, stress and total psychological distress. The details are given in Table No.2.

Table No.2 Significance of differences among the groups in their level of depression, anxiety, stress and total psychological distress as revealed in the Duncan test

\begin{tabular}{|c|c|c|c|c|c|c|}
\hline Variable & Sl. No & Group & $\begin{array}{l}\text { Mean } \\
\text { Score }\end{array}$ & 1 & 2 & 3 \\
\hline \multirow{3}{*}{ Depression } & 1 & Cvd & 16.5600 & ( ) & $*$ & $*$ \\
\hline & 2 & Cancer & 10.2667 & .. & ( ) & $*$ \\
\hline & 3 & Normals & 6.8400 &.. & .. & $($ ) \\
\hline \multirow{3}{*}{ Anxiety } & 1 & Cvd & 9.4667 & ( ) & $*$ & $*$ \\
\hline & 2 & Cancer & 7.7067 & .. & ( ) & .. \\
\hline & 3 & Normals & 7.2267 &.. & ( ) & .. \\
\hline \multirow{3}{*}{ Stress } & 1 & Cvd & 10.6400 & ( ) & $*$ & $*$ \\
\hline & 2 & Cancer & 12.5067 & .. & ( ) & $*$ \\
\hline & 3 & Normals & 14.4000 & .. & .. & ( ) \\
\hline \multirow{3}{*}{$\begin{array}{c}\text { Total } \\
\text { psychological } \\
\text { distress }\end{array}$} & 1 & $\mathrm{Cvd}$ & 36.4000 & ( ) & $*$ & $*$ \\
\hline & 2 & Cancer & 30.4800 &.. & $(\mathrm{)}$ &.. \\
\hline & 3 & Normals & 28.5600 & .. & ( ) & .. \\
\hline
\end{tabular}

Note:* Indicates significant difference between the groups compared 
The results obtained on Duncan test indicated that there were significant differences in their level of depression among cvd patients, cancer patients and normals. Cvd patients (16.5600) had significantly higher level of depression and normals (6.8400) had lower level of depression. Cancer patients (10.2667) had comparatively lower level of depression than cvd patients. Cvd patients (9.4667) had significantly higher level of anxiety and found to differ significantly from cancer patients and normals. Cancer patients (7.7067) and normals (7.2267) showed no significant differences in their level of anxiety.The results obtained on Duncan test indicated that there were significant differences in their level of stress among cvd patients, cancer patients and normals. Normals (14.4000) had higher level of stress than the patient groups. Cvd patients (10.6400) had significantly lower level of stress than cancer patients (12.5067).Cvd patients had significantly higher level of total psychological distress (36.4000) and found to differ significantly from cancer patients and normals. Cancer patients (30.4800) and normals (28.5600) showed no significant differences in their level of total psychological distress. The results revealed that cardiovascular patients had significantly higher level of depression, anxiety and total psychological distress than cancer patients and normals. The following studies supported our studies:

Depression is a common and often debilitating reaction to chronic illness. Upto one third of all medical in patients with chronic disease report at least moderate symptoms of depression, and lip to one quarter suffer from severe depression (Moody, Mc. Cormick and Williams, 1990., Rodin and Voshart, 1986). Depression is common among stroke patients, cancer patients and heart disease patients, as well as for those suffering from many other chronic diseases (Taylor and Aspinwall, 1990). Depression exacerbates the risk and course of several chronic disorders, most notably coronary heart disease (Anstey and Luszcz, 2002). Depressive symptoms are also associated with indicators of the metabolic syndrome, which may represent a related pathway to disease (Mc. CaffreyNiaura, Todaro, Swann and Carmelli, 2003).

Musselman and Nemeroff (2000) revealed a strong association between CHD and depression. Research also supports a strong link between depression and the likelihood of a heart attack, between depression and heart failure among the elderly and between hopeless and heart attack (Everson et al., 1996). Depression has been tied to inflammatory processes and hostility. Depression is strongly related to elevated c reactive protein, a marker of low grade systematic inflammation. Inflammatory processes appear to explain the relation of depression to heart failure (Pasic, Levy, Sullivan, 2003) and atherosclerosis (Danner, Kasl, Abramson and Vaccarina, 2003).

Among women, depression is a risk factor for metabolic syndrome, a precursor of heart disease (Kinder, Carnethon, Palaniappan, King and Fortmann, 2004). Women experience more anxiety after a heart attack than men do, which may go unrecognized or untreated. Female cvd patients experience poorer quality of life than men do (Emery et al., 2005). Acute stress, negative emotions, and sudden bursts of activity can precipitate sudden clinical events such as heart attack that lead to diagnosed disease. Stress and anxiety may be linked to heart disease via. changes in blood coagulation and fibrinolytic activity (Von Kaenel, Mills, Faiman and Dimsdale, 2001).

Myocardial Infarction (MI) patients who are depressed while in the hospital are less likely to be back at work 1 year later and more likely to be re-hospitalised compared with those patients who were not depressed (Stern, Pascale and Ackerman, 1977). Depression as well as anxiety, is so prevalent among chronically ill patients that many experts recommended routine screening for these symptoms during medical visits (Lowe et al., 2004).

IvonneLesman-Leegte (2006) found that the greatest proportion of psychological distress (10\%) was estimated among participants with self-reported chronic heart failure. They completed baseline data of 1050 elderly hospitalized patients with a confirmed diagnosis of Heart Failure. Almost $40 \%$ of the participants reported depressive symptoms.

Even though, the cancer patients in the study showed comparatively lower level of negative feelings than cvd patients, they too suffer from the depression, anxiety, stress and total psychological distress. The cancer patients who tend suppress their negative feelings such as depression, anxiety, distress which may only aggravate their illness. This may be the reason for their comparatively lesser level of depression, anxiety and total psychological distress than cvd patients. Cancer patients have significantly higher level of depression and total psychological distress than normals while they have significantly higher level of stress than cvd patients.

Cancer takes a substantial toll, both physically and psychologically. The physical difficulties usually stem from the pain and discomfort cancer can produce, particularly in the advancing and terminal phases of illness. Cancer can lead to down regulation of the immune system, which may enhance vulnerability to a variety of other disorders, including respiratory tract infections. These persistent health problems can compromise quality of life (Andersen, Kiecolt - Glaser and Glaser, 1994). Fatigue due to illness and treatment is also one of little main complaints of cancer patients (Andrykowski, Curran Lightner, 1998). Difficulties also arise as a consequence of treatment. Some cancers are treated surgically. Removal of organs can create cosmetic problems, as for patients with breast cancer who may have a breast removed (mastectomy) or for patients with head and neck cancer who may have a portion of this area removed, which leads to distorted body image. Body image concerns stem not only from about appearance following surgery but also from concerns about a sense of 
wholeness, bodily integrity and the ability to function normally. Either concern can complicate reactions to treatment (Carver et al., 1998). Some cancer patients receive debilitating follow up treatments. Patients undergoing chemotherapy may expect and experience debilitating nausea and vomiting and may develop anticipatory nausea and vomiting that occurs even before the chemotherapy session begins. As a result, symptoms of nausea, distress and vomiting can continue to adversely affect quality of life among cancer patients long after the treatment has ended (Montgomery and Bovbjerg, 2002).

Intermittent and long term depression are among the most common difficulties experienced as a result of cancer. Depression not only compromises quality of life in its own right, but it can have adverse effects on physical health outcomes as well, including the progression of cancer (Andersen et al., 1994). Depression is implicated in the progression of cancer, both by itself or in conjunction with other risk factors. Research has found a positive association between depression and cancer (Carney, Jons, Woolson, Noyes Doebbeling, 2003., Persky, Kempthrone, Rawson and Shekette, 1987). Depression can be associated with elevated neural endocrine responses such as cortisol and norepinephrine, which may inturn have implications for cancer via their impact on the immune system. Although cancer patients do not have more psychological distress than people without cancer for the most part, they are more susceptible to depression (Van'tspijker, Trijsburg and Duivenvoorden, 1997). Modest relationships between cancer development and use of denial or repressive coping strategies have been found as well (McKenna, Zevon, Corn and Rounds, 1999).

A few prospective studies in humans have found evidence of a link between uncontrollable stress and cancer (McKenna et al, 1999., Sklar and Anisman, 1981). Psychosocial variables may indirectly affect the initiation of cancer through consumption of a fatty diet or exposure to stress (Levy, 1983). Psychological stress appears to adversely affect the ability of NK cells to destroy tumours (Glaser, Rice, Speicher, Stout and Kiecolt - Glaser, 1986), an important finding because NK cell activity appears to be important in survival rates for certain cancers, especially early breast cancer (Levy, Herberman, Lipman, D' Angleo and Lee, 1991). All these studies supported our findings.

The normals were seemed to have more level of stress than cvd and cancer patients. The modern hectic and competitive world may make a normal person more prone to stress. Stress and distress were seen to be experienced by all people in the modern hectic life style.

\section{Conclusion}

It is hoped that the outcome of this study will lead to identify the level of psychological distress of cvd and cancer patients. The high prevalence and associated increase in morbidity and mortality justifies future research regarding the management of depression, anxiety and stress in both cardiovascular diseases and cancer. Effective distress management techniques can then be taken up to improve the well-being of those who suffering from these life style diseases. When the relevance of the present study is considered, it can be said that all the findings of the present investigation can be of much help for cardiovascular disease patients and cancer patients, and others directly or indirectly related to the welfare of cardiovascular disease patients and cancer patients. Whoever may be the persons using the findings of this study, the final beneficiaries will be the cardiovascular disease and cancer patients themselves. If they are aware of the risk factors of their illness, life style modification techniques and interventions to reduce their psychological distress, they will benefit from it. The role of psychological factors in the incidence and prognosis of a disease is explored. It is found that psychological factors have a significant role in the incidence and prognosis of cardiovascular disease and cancer. These findings can be immense value for cardiologists, oncologists, psychologists and counselors. They can initiate steps so that the cardiovascular disease patients and cancer patients will undergo the intervention strategies for life style modification and psychological distress reduction techniques, with the support of all who are concerned about the future of the patients. In order to be more beneficial to common people, a general awareness about the risk factors leading to cardiovascular disease and cancer along with its prevention and management has to be created by means of mass communication. For this purpose, services of print / electronic media will play a significant role. In addition, to this, regular medical camps / awareness programs can be organized with the help of various clubs such as Lions / Rotary etc. Regular visits by social workers / counselors to old age homes, and other social functions will be useful for educating the people about cardiovascular diseases and cancer. As the life style diseases especially cardiovascular patients and cancer are increasing; cardiologist, oncologists and psychologists and major hospitals will have to put in more efforts to organize awareness programs in a phased manner. It is suggested that major hospitals should make earnest endeavor to set up special departments consisting cardiologists, oncologists and psychologists solely for this program. 


\section{References:}

[1]. Andersen, B. L., Kiecolt-Glaser, J. K., and Glaser, R. (1994).A biobehavioral model of cancer stress and disease course. American Psychologist 49(5), 389-404.

[2]. Andrykowski, M. A., Curran, S. L., \&Lightner, R. (1998). Off-treatment fatigue in breast cancer survivors: A controlled comparison. Journal of Behavioral Medicine, 21, 1-18.

[3]. Anstey, K. J.,\&Luszcz, M. A. (2002). Mortality risk varies according to gender and change in depressive status in very old adults. Psychosomatic Medicine, 64, 880-888.

[4]. Baum, A., Grunberg, N.E. (1991). Gender, stress, and health.Health psychology, 10(2), 80-5.

[5]. Carney., Caroline, P., Laura Jones., Robert, F, Woolson., Russell Noyes., Bradley, N, Doebbeling. (2003). Relationship between depression and pancreatic cancer in the general population.Psychosomatic medicine, 65(5),884-8.

[6]. Carver, C. S., Pozo-Kaderman, C., Price, A. A., Noriega, V., Harris, S. D., Derhagopian, R. P., et al. (1998). Concerns about aspects of body image and adjustment to early stage breast cancer. Psychosomatic Medicine, 60, 168-174.

[7]. Chochinov, H.M., Hack, T., Hassard, T. (2005). Dignity therapy: a novel psychotherapeutic intervention for patients nearing death. J ClinOncol, 23, 5520-5525.

[8]. Danner, M., Kasl, S.V., Abramson, J.L., Vaccarino, V.(2003). Association between depression and elevated C-reactive protein.Psychosom Med, 65(3), 347-56.

[9]. Emery Krogh, R.W., Arom, K.V. (2005). Patient-prosthesis mismatch: Impact on patient survival and valve related events: a 25year experience with the St. Jude Medical valve prosthesis. Presented at the Society of Thoracic Surgeons at the 41st Annual Meeting, San Antonio, TX

[10]. Everson, S.A., Goldberg, D.E., Kaplan, G.A., Cohen, R.D., Pukkula, E., Tuomilehto, J., \&Salonen, J.T. (1996). Hopelessness and risk of mortality and incidence of myocardial infraction and cancer.Psychosomatic Medicine, 58, 113-121.

[11]. Glaser, R., Rice, J., Speicher, C.E., Stout, J.C., Kiecolt-Glaser, J.K. (1986). Stress depresses interferon production concomitant with a decrease in natural killer cell activity. Behavioral Neuroscience, 100, 675-678.

[12]. IvonneLesman-Leegte., Tiny Jaarsma., Dirk Jan van Veldhuisen.(2006). Psychological distress and cardiovascular disease.European heart journal, 27(9), 1123.

[13]. J. M. McCaffery, R. Niaura, J. F. Todaro, G. E. Swan, and D. Carmelli. (2003). "Depressive symptoms and metabolic risk in adult male twins enrolled in the National Heart, Lung, and Blood Institute Twin Study," Psychosomatic Medicine, vol. 65, no. 3, pp. 490497.

[14]. Kesteloot, H., Sans, S., Kromhout, D. (2006). Dynamics of cardiovascular and all-cause mortality in Western and Eastern Europe between 1970 and 2000.Eur Heart J, 27, 107-113.

[15]. Kinder, L.S., Carnethon, M.R., Palaniappan, L.P., King, A.C., Fortmann, S.P. (2004). Depression and Metabolic Syndrome in Young Adults: Findings from the Third National Health and Nutrition Examination Survey.Psychosomatic Medicine, 66(3), 316-22.

[16]. Lecrubier, Y. (2001). The influence of comorbidity on the prevalence of suicidal behaviour.European psychiatry: the journal of the Association of European Psychiatrists, 16(7), 395-9

[17]. Levi, S.M. (1983). "Giving It Up - At the Cellular Level".Science News, 12: 124-148

[18]. Levy, S.M., Herberman, R.B., Lippman, M., D’Angelo, T., Lee, J. (1991). Immunological and psychosocial predictors of disease recurrence in patients with early stage breast cancer. Behavioral Medicine.Summer, 67-75.

[19]. Löwe B, Spitzer RL, Gräfe K, Kroenke K, Quenter A, Zipfel S, et al. (2004). Comparative validity of three screening questionnaires for DSM-IV depressive disorders and physicians' diagnoses.Journal of Affective Disorders.;78:131-140.

[20]. Matthews, K.A, Shumaker, S.A, Bowen, D.J, et al (1997). Women's Health Initiative. Why now? What is it? What's new? Am Psychol., 52, 101-16.

[21]. McKenna, M. C., Zevon, M. A., Corn, B., \&Rounds, J. (1999). Psychological factors and the development of breast cancer: A metaanalysis. Health Psychol, 18, 520-531.

[22]. Michaela Kreuzer.,Joachim Heinrich., LotharKreienbrock., Angelika Schaffrath Rosario.,; Michael Gerken., H. Erich Wichmann. (2002). Risk factors for lung cancer among non-smoking women.International Journal of Cancer, 100 (6), 706-713,

[23]. Montgomery, G.H, Weltz, C.R., Seltz, G., Bovbjerg, D.H. (2002). Brief pre-surgery hypnosis reduces distress and pain in excisional breast biopsy patients. International Journal of Clinical and Experimental Hypnosis, 50, 17-32

[24]. Moody, L., McCormick, K., Williams, A. (1990). Disease and symptom severity, functional status, and quality of life in chronic bronchitis and emphysema.J Behavioral Medicine.,13, 297-304.

[25]. Musselman.,\& B. Nemeroff. (1998). The Relationship of Depression to Cardiovascular Disease: Epidemiology, Biology, and Treatment. Arch Gen Psychiatry., 55, 580-592.

[26]. Pasic, J., Levy, W., Sullivan, M (2003). Cytokines in depression and heart failure. Psychosomatic Medicine, 65, 181-193.

[27]. Persky, V.W, Kempthorne-Rawson, J., Shekelle, R.B. (1987). Personality and risk of cancer: 20 year follow up of the Western Electric study. Psychosomatic Med, 49, 439-449.

[28]. Rodin, G., \&Voshart, K. (1986) Depression in the medically ill: an overview. American Journal of Psychiatry, 143, 696-705.

[29]. Sklar, L.S., \&Anisman, H. (1981).Stress and cancer. Psychological Bulletin, 89, 369-406

[30]. Stern, M. J., Pascale, L., \& Ackerman, A. (1977). Life adjustment post myocardial infarction: Determining predictive variables. Archives of Internal Medicine, 137, 1680-1685.

[31]. Taylor, S.E, Klein, L.C., Lewis, B.P., Gruenewald, T.L., Gurung, R.A., Updegraff, J.A. (2000). Bio-behavioral responses to stress in females: tend-and-befriend, not fight-or-flight. Psychol Rev., 107(3), 411-29.

[32]. Taylor, S.E., \&Aspinwall, L.G. (1990).Psychosocial aspects of chronic illness. In P.T. Costa, Jr., \& G.R. VandenBos (Eds.), Psychological aspects of serious illness: Chronic conditions, fatal disease, and clinical care (pp. 3-60). Washington, DC: American Psychological Association.

[33]. Van'tSpijker, A., Trijsburg, R.W., Duivenvoorden, H.J. (1997). Psychological sequelae of cancer diagnosis: a meta-analytical review of 58 studies after 1980. Psychosom Med., 59, 280-293.

[34]. vonKänel R, Mills P, Dimsdale J (2001) Short-term-hyperglycemia induces lymphopenia and lymphocyte subset redistribution. Life Sciences 69: 255-262. Impact factor: 2.158

[35]. Wing, R R., Matthews, K A., Kuller, L H., Meilahn, E N., Plantinga, P L .(1991). Weight gain at the time of menopause.Archives of internal medicine, 151(1), 97-102.

[36]. Yohannes AM, Willgoss TG, Baldwin RC, Connolly MJ.(2010). Depression and anxiety in chronic heart failure and chronic obstructive pulmonary disease: prevalence, relevance, clinical implications and management principles. International Journal of Geriatric Psychiatry, 25(12):1209-21. doi: 10.1002/gps.2463.

[37]. Zabora, J., Blanchard, C., Smith, E., Roberts, C., Glajchen, M., Sharp, J., et al. (2001).Prevalence of psychological distress among cancer patients across the disease continuum.Journal of Psychosocial Oncology, 15(2), 73-87. 